\title{
Proton Pump Inhibition Enhances the Cytotoxicity of Paclitaxel in Cervical Cancer
}

Taejong Song, MD, $\mathrm{PhD}^{1}$
Hye-Kyung Jeon, $\mathrm{MSc}^{2}$
Ji Eun Hong, MSc
Jung-Joo Choi, $\mathrm{MSc}^{2}$
Tae-Joong Kim, MD, $\mathrm{PhD}^{2}$
Chel Hun Choi, MD, $\mathrm{PhD}^{2}$
Duk-Soo Bae, MD, $\mathrm{PhD}^{2}$
Byoung-Gie Kim, MD, PhD²
Jeong-Won Lee, MD, $\mathrm{PhD}^{2}$

${ }^{1}$ Department of Obstetrics and Gynecology, Kangbuk Samsung Hospital, Sungkyunkwan University School of Medicine, Seoul, ${ }^{2}$ Department of Obstetrics and Gynecology, Samsung Medical Center, Sungkyunkwan University School of Medicine, Seoul, Korea

Correspondence: Jeong-Won Lee, MD, PhD Department of Obstetrics and Gynecology, Samsung Medical Center, Sungkyunkwan University School of Medicine, 81 Irwon-ro, Gangnam-gu, Seoul 06351, Korea

Tel: 82-2-3410-1382

Fax: 82-2-3410-0630

E-mail: garden.lee7@gmail.com

Co-correspondence: Byoung-Gie Kim, MD, PhD Department of Obstetrics and Gynecology, Samsung Medical Center, Sungkyunkwan University School of Medicine, 81 Irwon-ro, Gangnam-gu, Seoul, 06351, Korea

Tel: 82-2-3410-3513

Fax: 82-2-3410-0630

E-mail: bgkim@skku.edu

Received January 21, 2016

Accepted September 11, 2016

Published Online September 27, 2016

*Taejong Song and Hye-Kyung Jeon contributed equally to this work.

\section{Purpose}

This study was conducted to investigate whether a proton pump inhibitor (PPI) could enhance chemosensitivity via the inhibition of vacuolar-type $\mathrm{H}^{+}$ATPase (V-ATPase) in cervical cancer.

\section{Materials and Methods}

The expression of V-ATPase was evaluated in 351 formalin-fixed, paraffin-embedded human cervical cancer tissues using immunohistochemistry and compared with clinicopathologic risk factors for disease prognosis. The influence of cell proliferation and apoptosis following V-ATPase siRNA transfection or esomeprazole pretreatment was assessed in cervical cancer cell lines using 3-(4,5-dimethylthiazol-2-yl)-2,5-diphenyl tetrazolium bromide and enzymelinked immunosorbent assay, respectively.

\section{Results}

Immunohistochemical analysis revealed that V-ATPase was expressed in about $60 \%$ of cervical cancer tissue samples (211/351), and the expression was predominantly found in adenocarcinoma histology $(p=0.016)$. Among patients with initially bulky cervical cancer $(n=89)$, those with V-ATPase expression had shorter disease-free survival $(p=0.005)$ and overall survival $(p=0.023)$. Co-treatment with V-ATPase siRNA or esomeprazole with paclitaxel significantly decreased the cell proliferation of cervical cancer cell lines, including HeLa and INT407, compared to cell lines treated with paclitaxel alone $(p<0.01)$. Moreover, V-ATPase siRNA or esomeprazole followed by paclitaxel significantly increased the expression of active caspase- 3 in these cells compared to cells treated with paclitaxel alone (both, $p<0.05$ )

\section{Conclusion}

V-ATPase was predominantly expressed in cervical adenocarcinoma, and the expression of V-ATPases was associated with poor prognosis. The inhibition of V-ATPase via siRNA or PPI (esomeprazole) might enhance the chemosensitivity of paclitaxel in cervical cancer cells.

\section{Key words}

Uterine cervical neoplasms,

Vacuolar proton-translocating ATPases,

Proton pump inhibitors, Esomeprazole, Small interfering RNA, Antineoplastic agents 


\section{Introduction}

Cancer of the uterine cervix is the second most common malignancy in women worldwide [1]. Although it is accepted that early-stage cervical cancer is curable with radical surgery, radiotherapy, or chemoradiation, the prognosis in patients with bulky tumors or adenocarcinoma histology is relatively poor $[2,3]$. Moreover, although chemotherapy for advanced-stage or recurrent cervical cancer has been applied, it is not effective and has merely a palliative purpose because of chemoresistance [4]. Therefore, a new therapeutic strategy for these patients to overcome chemoresistance in such patients is urgently needed.

One of major mechanisms of chemoresistance is increased acidification of the tumor microenvironment. Proton pumps such as vacuolar type $\mathrm{H}^{+}$-ATPase (V-ATPase) are major regulators of cellular $\mathrm{pH}$ [5]. V-ATPases pump protons across the plasma membrane and across the membranes of a wide array of intracellular compartments [5]. Some human tumor cells, particularly those selected for multidrug resistance, exhibit enhanced V-ATPase activity [6]. Therefore, treatment with proton pump inhibitor (PPI; esomeprazole), which directly inhibits V-ATPase at the cellular level [7,8], may be an option for reversing multidrug resistance. Here, we investigated whether PPI increased the sensitivity of tumor cells to cytotoxic agents via inhibition of V-ATPase in cervical cancer.

\section{Materials and Methods}

\section{Tumor samples}

This study was reviewed and approved by the Institutional Review Board at Samsung Medical Center, Seoul, Republic of Korea. A total of 351 patients with cervical cancer who underwent type I-III radical hysterectomy with or without pelvic/paraaortic lymph node dissection at Samsung Medical Center between February 2002 and May 2009 were included in this study. Patient inclusion criteria were (1) availability of tissue samples for tissue microarray construction and (2) early-stage cervical cancer that were candidates for surgery because pathological findings provide the most accurate information about tumor volume, depth of tumor invasion, and lymph node metastasis. Cervical cancer patients with the pathology of sarcoma, malignant melanoma, or neuroendocrine carcinoma, including small cell carcinoma, were excluded. After surgery, patients received adjuvant treatment according to their pathological findings. Patients then underwent follow-up examinations approximately every three months for the first 2 years, every 6 months for the next 3 years, and every year thereafter. Disease-free survival was defined as the period between initial treatment and relapse. Overall survival was measured from the time of initiation of therapy to the time of death or the date of final contact. Data for patients who had not experienced relapse or death were censored as of the date of the final observation.

\section{Immunohistochemical analysis}

All hematoxylin and eosin-stained slides were reviewed and representative tumor tissue samples were selected. The corresponding formalin-fixed paraffin-embedded tissue blocks were retrieved and the selected area was circled on the slide with a marker pen for tissue microarray construction. Each 6.0-mm tissue core was selected from the representative region of each paraffin block using the AccuMax (IsuAbxis, Seoul, Korea) as described previously [9]. For immunohistochemical studies, tissue microarray blocks were sectioned into 4 - $\mu \mathrm{m}$-thick slices. The primary antibody used was rabbit polyclonal V-ATPase subunit C1 antibody (Santa Cruz Biotechnology, Santa Cruz, CA). Tissue sections were deparaffinized three times in xylene for a total of 15 minutes and subsequently rehydrated. Immunostaining for V-ATPase was performed using a Bond-max automated immunostainer (Leica Biosystems, Melbourne, Australia) and the Bond Polymer Refine Detection Kit (Vision Biosystems, Melbourne, Australia). Briefly, antigen retrieval was conducted at $97^{\circ} \mathrm{C}$ for 20 minutes in ER1 buffer. After blocking the endogenous peroxidase activity with $3 \%$ hydrogen peroxidase for 10 minutes, primary antibody incubation was conducted for 15 minutes at room temperature at an antibody dilution of 1:200. Negative controls (substitution of primary antibody for Tris-buffered saline [TBS]) were run simultaneously. The immunostaining was evaluated independently by two pathologists who were blinded to patient outcome. A previously described scoring method $[10,11]$ was used for the evaluation of V-ATPase expression. A case was considered positive when more than $1 \%$ of the epithelial cells in 10 random, high-power fields were positively stained.

\section{Cell lines}

Human cervical cancer cell lines (HeLa, SiHa, ME180, MS751, and CaSki) were obtained from the American Type Culture Collection (Manassas, VA). The INT407 cell line was obtained from the European Collection of Cell Culture (Salisbury, UK). The HeLa cell line was cultured in Dulbecco's modified Eagle's medium (Gibco BRL, Rockville, MD), the INT407, SiHa, ME180, and MS751 cell lines were cultured in 
MEM (Gibco BRL); and the CaSki cell line was cultured in RPMI (Gibco BRL). All media were supplemented with 10\% fetal bovine serum (FBS; Sigma-Aldrich, St. Louis, MO) and $0.1 \%$ penicillin-streptomycin (Sigma-Aldrich). All cells were grown at $37^{\circ} \mathrm{C}$ in a humidified $5 \% \mathrm{CO}_{2}$ atmosphere.

\section{Western blot analysis}

For analysis of V-ATPase expression, cells were lysed in PRO-PRE Protein Extraction Solution (Intron Biotechnology, Seongnam, Korea). Protein concentration was determined using a BCA protein kit (Thermo Scientific, Rockford, IL). Cell lysates (50 $\mu \mathrm{g}$ of total protein) were separated in $12 \%$ acrylamide gels by sodium dodecyl sulfate-polyacrylamide gel electrophoresis and then transferred to Immobilon PVDF membrane filter paper (Millipore, Billerica, MA). Membranes were blocked with 5\% skim milk in TBS containing 0.1\% Tween-20 for 1 hour at room temperature. Protein bands were probed with V-ATPase subunit $C 1$ antibody at a 1:1,000 dilution (Santa Cruz Biotechnology), tubulin and glyceraldehyde 3-phosphate dehydrogenase antibody at a 1:3,000 dilution (Epitomics, Burlingame, CA), and then labeled with horseradish peroxidase-conjugated anti-rabbit antibody (GE Healthcare, Piscataway, NJ) and anti-goat antibody (Santa Cruz Biotechnology). Bands were visualized by enhanced chemiluminescence using an ECL kit (Amersham Biosciences, Buckinghamshire, UK) according to the manufacturer's protocol.

\section{Small interfering RNA transfection and drug treatment}

V-ATPase V1C1 small interfering RNA (siRNA) and negative control siRNA were obtained from Santa Cruz Biotechnology. Cells were seeded at $5 \times 10^{3}$ cells / well in a 96-well microplate in RPMI 1640 with 10\% FBS. All siRNAs were transfected into the cells using Lipofectamine 2000 (Invitrogen, San Diego, CA) according to the manufacturer's protocol. After 24 hours of siRNA transfection, cells were treated with various concentrations of paclitaxel (Sigma-Aldrich) and then incubated at $37^{\circ} \mathrm{C}$ for 48 hours. Esomeprazole (AstraZeneca, Mölndal, Sweden) was resuspended in normal saline at a concentration of $5 \mathrm{mg} / \mathrm{mL}$. Cells were seeded at $3 \times 10^{3}$ cells/well in a 96-well microplate in RPMI 1640 with $10 \%$ FBS. Cells were pretreated or not with esomeprazole (20-30 mg/mL) based on the protocols in previous reports $[12,13]$. After 24 hours of treatment, cells were treated with various concentrations of paclitaxel and incubated at $37^{\circ} \mathrm{C}$ for 48 hours.

\section{3-(4,5-Dimethylthiazol-2-yl)-2,5-diphenyl tetrazolium bromide assay}

3-(4,5-Dimethylthiazol-2-yl)-2,5-diphenyl tetrazolium bromide (MTT) assay is based on the conversion of MTT to insoluble MTT-formazan by cleavage of the tetrazolium ring by mitochondrial dehydrogenase enzymes of living cells. MTT solution (Amresco, Solon, $\mathrm{OH}$ ) was subsequently added to each well. After 4 hours of additional incubation, the medium was discarded, $100 \mu \mathrm{L}$ of acidic isopropanol $(0.1$ $\mathrm{N} \mathrm{HCl}$ in absolute isopropanol) was added, and the plate was shaken gently. The absorbance at a wavelength of 540 $\mathrm{nm}$ was recorded by using a Falcon microplate reader (Becton Dickinson Labware, Franklin Lakes, NJ) and the percentage viability was calculated as follows: (optical density [OD] of drug-treated sample/control OD) $\times 100$. Additionally, the mean percent viability was calculated from cytotoxicity experiments at different paclitaxel concentrations that showed a significant difference from the control $(\mathrm{p}<0.05)$. Each sample was assayed in triplicate, and the experiment was repeated three times.

\section{Enzyme-linked immunosorbent assay}

For analysis of active caspase-3 expression, cells were lysed in PRO-PRE Protein Extraction Solution (Intron Biotechnology). The protein concentration was determined using a BCA protein kit (Thermo Scientific). Enzyme-linked immunosorbent assay (ELISA) kits were used according to the manufacturer's instructions to measure concentrations of active caspase-3 (Invitrogen). All samples were measured in triplicate.

\section{Measurement of intracellular $\mathrm{pH}$ change}

The change in intracellular $\mathrm{pH}$ was measured as previously described [14]. Briefly, cells grown as a monolayer on a $35 \mathrm{~mm}$ confocal dish (\#200350, SPL Lifescience, Pocheon, Korea) were loaded with $1 \mu \mathrm{g} / \mathrm{mL}$ BCECF-AM $\left(2^{\prime}, 7^{\prime}\right.$-bis(2-carboxyethyl)-5-(and-6)-carboxyfluorescein; Invitrogen) solution. The distribution of fluorescence was determined using a confocal microscope (LSM700, Zeiss, Jena, Germany) with excitation and emission wavelengths set to $488 \mathrm{~nm}$ and $525 \mathrm{~nm}$, respectively [15]. The intensity of the fluorescence signal was measured using a VICTOR2 plate reader (PerkinElmer, Boston, MA), and the values were corrected to cell numbers by MTT assay [16]. Each sample was assayed in triplicate, and the experiment was repeated three times. 


\section{Statistical analysis}

After using the Shapiro-Wilk test to confirm that the data were normally distributed, the Wilcoxon rank sum test was used to compare the median values, and the two-sample $t$ test was used to compare the mean values. The chi-square test or Fisher exact test was used to compare the frequency distributions between categorical variables. Disease-free survival and overall survival curves were evaluated with the Kaplan-Meier method and compared using the log-rank test. The Cox proportional hazards model was used to calculate the hazard ratio and the $95 \%$ confidential interval (CI). The SPSS software ver. 17.0 (SPSS Inc., Chicago, IL) was used for all statistical analyses. All $p$-values were two-sided and were considered statistically significant at $\mathrm{p}<0.05$.

\section{Results}

\section{Immunohistochemistry and its clinical significance}

We assessed V-ATPase expression using immunohistochemistry in tissue microarrays composed of 351 human cervical cancer tissues. V-ATPase was predominantly expressed in the cell membrane and cytoplasm, and representative results of immunohistochemical staining for V-ATPase are shown in Fig. 1A. Immunohistochemical analysis demonstrated that V-ATPase was expressed in $60.1 \%$ of cervical cancer tissue samples (211/351), and the expression rate of V-ATPase in cervical adenocarcinomas was $73.4 \%$ (Fig. 1B), which is significantly higher than that of squamous cell cervical cancer $(57.1 \%, \mathrm{p}=0.016)$. During the median follow-up period of 65.5 months, a total of 351 patients included in this study had a disease-free survival of $84.4 \%$ and overall survival of $95.0 \%$. When the disease-free survival of all 351 patients was analyzed based on the V-ATPase expression (S1 Table), V-ATPase was not correlated with disease-free survival. However, when the analysis was confined to 89 patients with bulky cervical tumor (defined as tumor diameter $>4 \mathrm{~cm}$ ), patients with V-ATPase expression had shorter disease-free survival $(\mathrm{p}=0.005)$ and overall survival $(\mathrm{p}=0.023)$ than those without V-ATPase expression (Fig. 1C, S2 Table). Moreover, when confined to 55 patients with bulky V-ATPase positive tumors, patients with adenocarcinoma had shorter disease-free survival than those with squamous cell carcinoma ( $\mathrm{p}=0.005$ ) (Fig. 1D).

\section{V-ATP V1C1 siRNA transfection significantly enhanced cytotoxicity of paclitaxel in cervical cancer cells}

We measured the basal expression of V-ATPase in various cervical cancer cell lines of HeLa, SiHa, MS751, INT407, and CaSki using Western blot. V-ATPase protein was expressed in most cervical cancer cell lines, with the exception of ME180 cells (Fig. 2A). Subsequently, in vitro experiments were performed to assess whether blocking V-ATPase by specific siRNA transfection enhanced the sensitivity to chemotherapy in cervical cancer cell lines. Based on our immunohistochemical results of V-ATPase expression, we used HeLa and INT407 cells, which were originally derived from cervical adenocarcinoma, for subsequent analyses. After HeLa and INT407 cells were transfected with V-ATPase siRNA or control siRNA, the expression of V-ATPase was determined by western blot analysis. V-ATPase expression was decreased at 48 hours after V-ATPase siRNA transfection in comparison to that in the controls (Fig. 2B), suggesting that V-ATPase expression was effectively down-regulated by the V-ATPase siRNA. We then assessed the effects of V-ATPase siRNA transfection on cell survival after treatment with cytotoxic drugs. As shown in Fig. 2C, pretreatment with V-ATPase siRNA significantly enhanced the cytotoxicity of paclitaxel in HeLa and INT407 cells compared with paclitaxel treatment alone. However, V-ATPase siRNA transfection did not enhance the cytotoxicity of cisplatin in HeLa cells or the cytotoxicity of paclitaxel in $\mathrm{SiHa}$ cells originating from squamous cervical cancer (S3 Fig.). To assess cellular apoptosis, active caspase-3 was measured using ELISA in HeLa and INT407 cells following treatment with paclitaxel with or without V-ATPase siRNA pretreatment. The results showed that V-ATPase siRNA transfection significantly enhanced the apoptotic activity of chemotherapy in HeLa and INT407 cells (Fig. 3).

\section{Esomeprazole pretreatment significantly increased the cytotoxicity of paclitaxel in cervical cancer cells}

Subsequently, in vitro studies using esomeprazole were repeated to assess whether esomeprazole pretreatment, such as blocking V-ATPase by specific siRNA transfection, enhanced cytotoxicity and apoptosis. The results showed that esomeprazole pretreatment significantly enhanced the cytotoxicity of paclitaxel in HeLa and INT407 cells compared with paclitaxel treatment alone (Fig. 4A). Interestingly, the effects of esomeprazole pretreatment in HeLa (37\%) and INT407 $(47 \%)$ cell lines derived from adenocarcinoma were more remarkable than those in $\mathrm{SiHa}(11 \%)$ and MS751 (18\%) derived from non-adenocarcinomas such as squamous cell carcinoma (S4 Fig.). The effects of esomeprazole pretreatment $(20 \mu \mathrm{g} / \mathrm{mL}$ concentration) on apoptosis also signifi- 
A
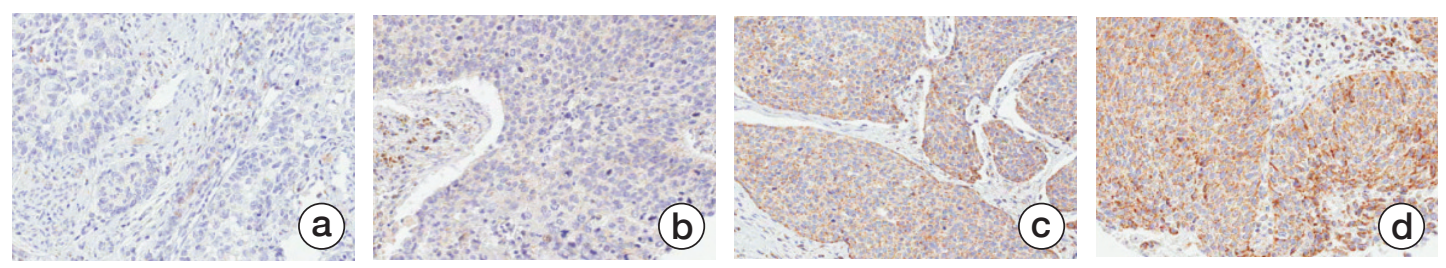

B

Expression of V-ATPase in cervical cancer according histology based on IHC $(\mathrm{n}=351)$

\begin{tabular}{lccc} 
& \multicolumn{2}{c}{ Staining extent, $\mathrm{n}(\%)$} & \multirow{2}{*}{ p-value } \\
\cline { 2 - 3 } Cervical specimen & Negative & Positive & \\
Squamous cell carcinoma $(\mathrm{n}=287)$ & $123(42.9)$ & $164(57.1)$ & 0.016 \\
Adenocarcinoma $(\mathrm{n}=64)$ & $17(26.6)$ & $47(73.4)$ & \\
\hline
\end{tabular}

C
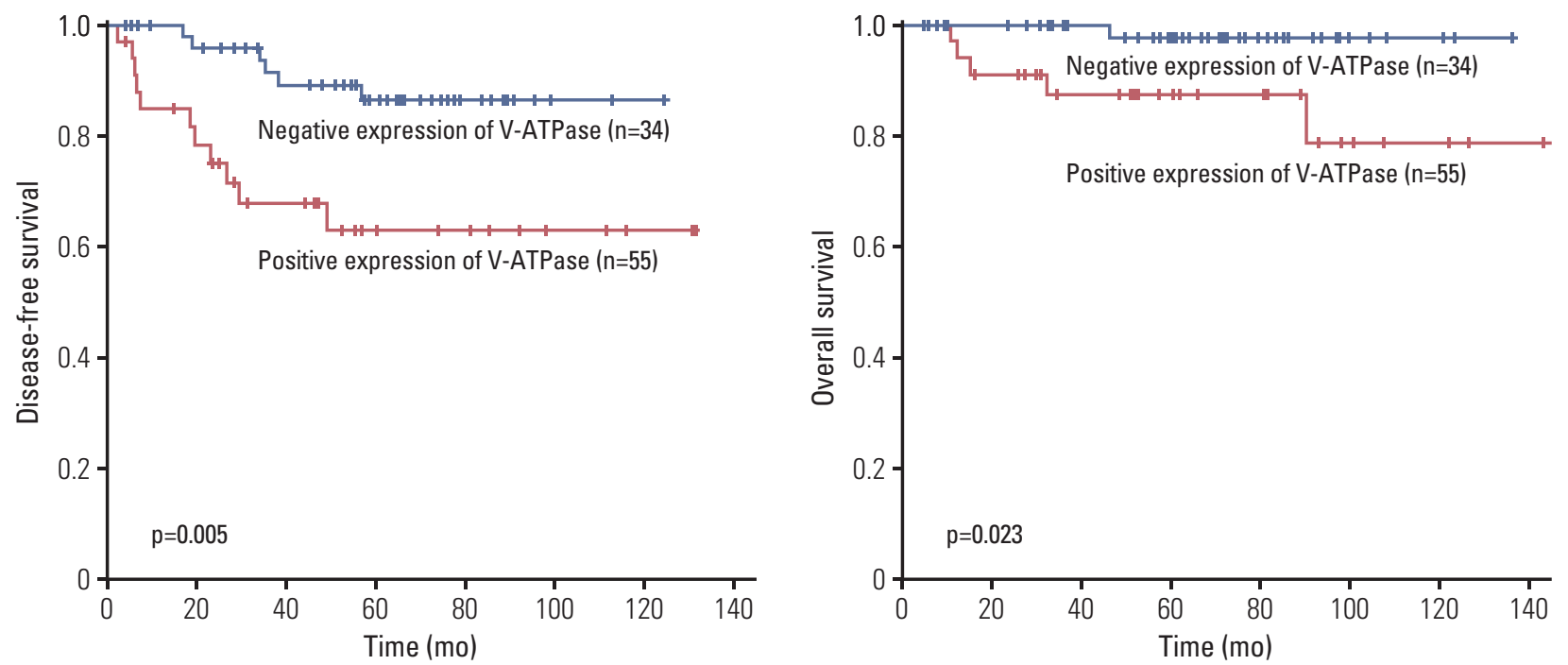

Fig. 1. Vacuolar-type $\mathrm{H}^{+}$ATPase (V-ATPase) was predominantly expressed in cervical adenocarcinoma, which was associated with poor prognosis. (A) Representative V-ATPase staining from cervical cancer (a, no staining; b, weak staining; , moderate staining; $d$, strong staining, $\times 400$ ). (B) Expression of V-ATPase in cervical cancer according to histology based on immunohistochemistry (IHC) ( $\mathrm{n}=351)$. (C) Kaplan-Meier curves showed disease-free survival and overall survival according to V-ATPase expression in 89 patients with bulky cervical cancers (tumor diameter $>4 \mathrm{~cm}$ ). (Continued to the next page)

cantly increased the expression of active caspase-3 in HeLa and INT407 cells when compared with paclitaxel treatment alone (Fig. 4B and C).

\section{Measurement of intracellular $\mathrm{pH}$ change}

To verify that esomeprazole treatment induces cytosolic acidification through inhibition of V-ATPase activity, we measured the changes in intracellular $\mathrm{pH}$ following esomeprazole treatment based on the intensity and distribu- 


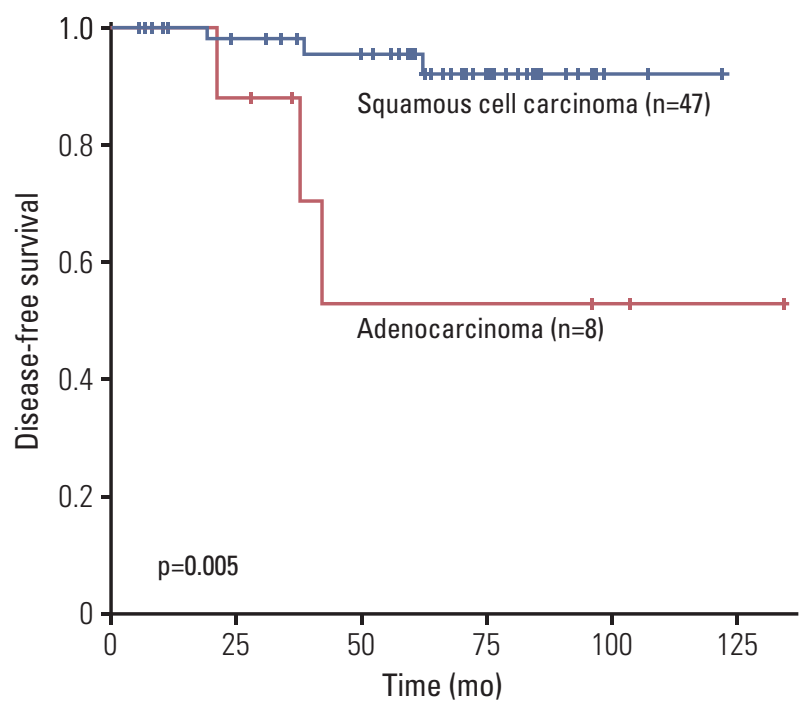

Fig. 1. (Continued from the previous page) (D) Kaplan-Meier curves showed disease-free survival according to cell type in 55 patients with bulky V-ATPase positive tumors.
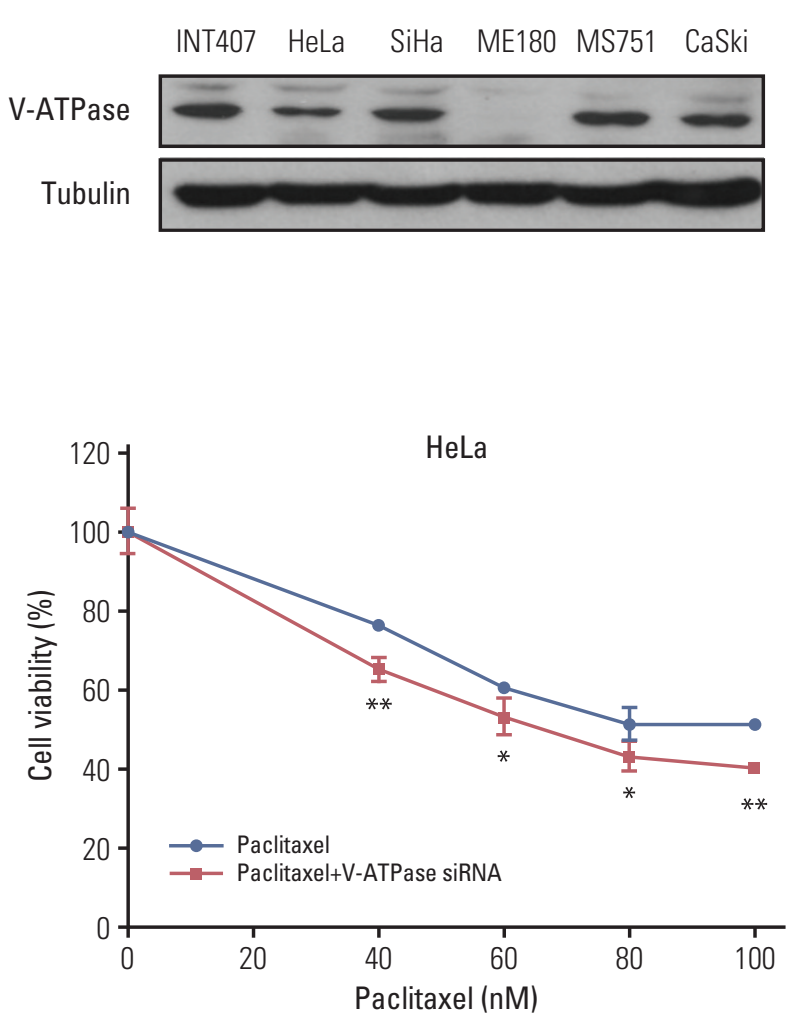

tion of BCECF using a confocal microscope (LSM700). We used HeLa cells that showed high expression of V-ATPase and were originally derived from cervical adenocarcinoma based on the results of earlier cell survival and apoptosis studies. Live-cell imaging of the BCECF distribution within the cells revealed that immunofluorescence staining after esomeprazole treatment was weak compared with that of no treatment, suggesting that esomeprazole treatment induced a decrease in intracellular pH (Fig. 5A). As shown in Fig. 5B, the fluorescence intensity ratio showed that intracellular $\mathrm{pH}$ decreased significantly after esomeprazole treatment in HeLa and INT407 cells treated with esomeprazole for 3 hours (both, $\mathrm{p}<0.01$ ).

\section{Discussion}

In this study, we found that the V-ATPase expression in cervical cancer was significantly increased in patients with

A

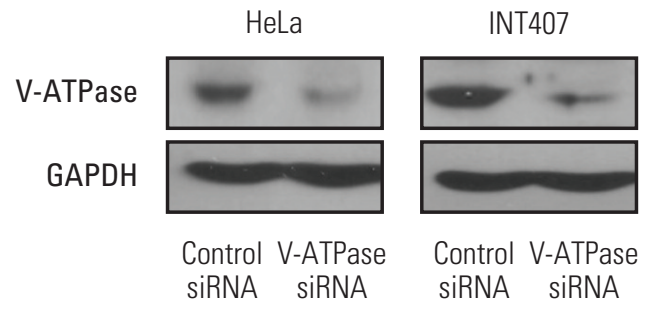

C

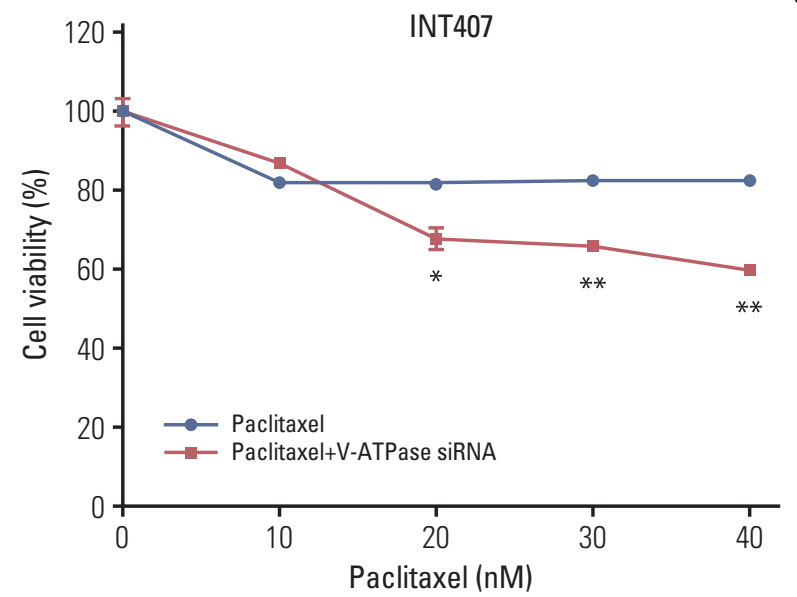

Fig. 2. (A) Expression of vacuolar-type $\mathrm{H}^{+}$ATPase (V-ATPase) in various cervical cancer cell lines. (B) Expression of V-ATPase was decreased by V-ATPase siRNA in HeLa and INT407 cells. (C) The effects of V-ATPase siRNA transfection on cell viability with paclitaxel in HeLa and INT407 cells. GAPDH, glyceraldehyde 3-phosphate dehydrogenase. * $<<0.05$, ${ }^{* *} \mathrm{p}<0.01$. 
HeLa

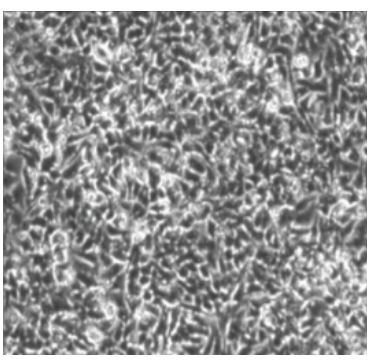

Control

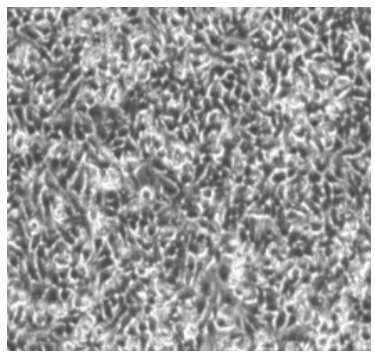

V-ATPase siRNA

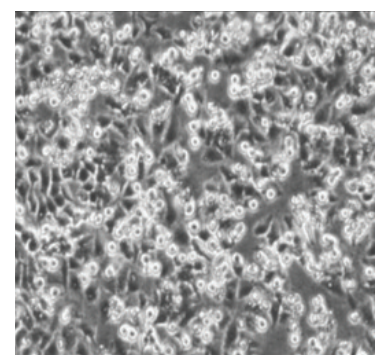

Paclitaxel

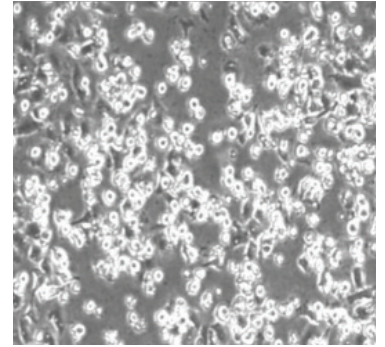

Paclitaxel+V-ATPase siRNA

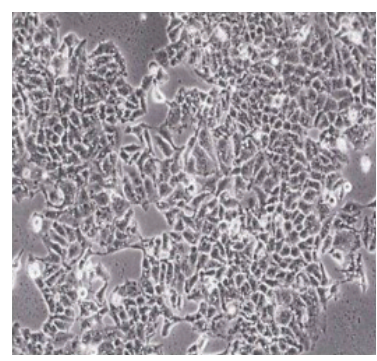

Control

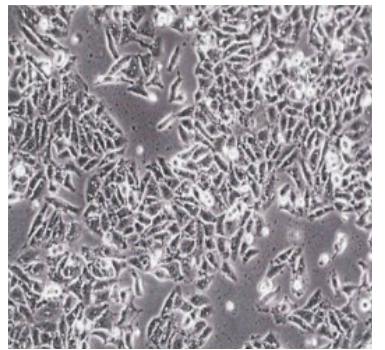

V-ATPase siRNA
INT407

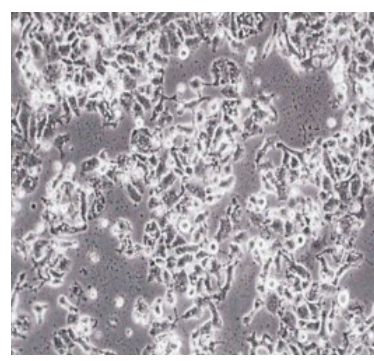

Paclitaxel

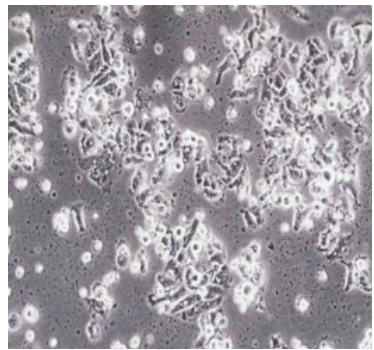

Paclitaxel+V-ATPase siRNA
HeLa

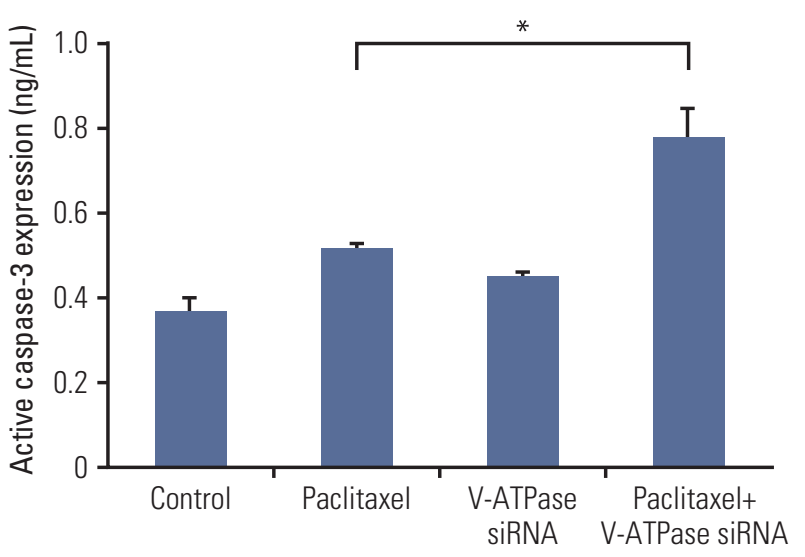

INT407

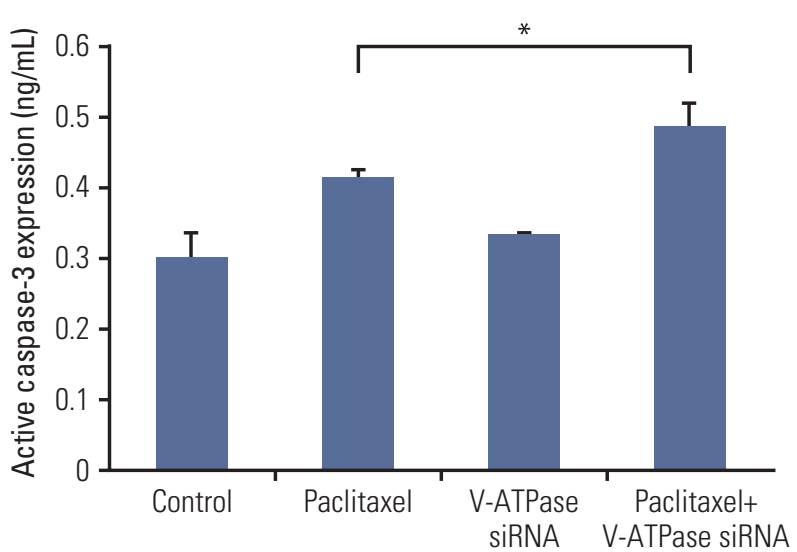

Fig. 3. Effects of vacuolar-type $\mathrm{H}^{+}$ATPase (V-ATPase) siRNA transfection on cell apoptosis with paclitaxel in HeLa and INT407 cells. (A) Cell death was observed by light microscopy in HeLa and INT407 cells ( $\times 100)$. (B) Expression of active caspase-3 was measured by enzyme-linked immunosorbent assay in HeLa and INT407 cells. * $\mathrm{p}<0.05$.

adenocarcinoma histology and was correlated with poor disease-free survival and overall survival in patients with bulky cervical tumor. Moreover, the inhibition of V-ATPase via PPI (esomeprazole) or its specific siRNA enhanced the cytotoxicity of paclitaxel in cervical cancer cells. To the best of our knowledge, this is the first study of V-ATPase in cervical cancer.
Bulky cervical tumors in early cervical cancer are known as prognostic factors for survival after surgery, and patients with bulky cervical tumors often receive additional adjuvant therapy or neoadjuvant chemotherapy [17]. In the present study, we also found that patients with bulky cervical tumors had a 1.21 hazard ratio for recurrence $(95 \%$ CI, 1.06 to 1.37$)$ (S1 Table), and V-ATPase expression was correlated with 

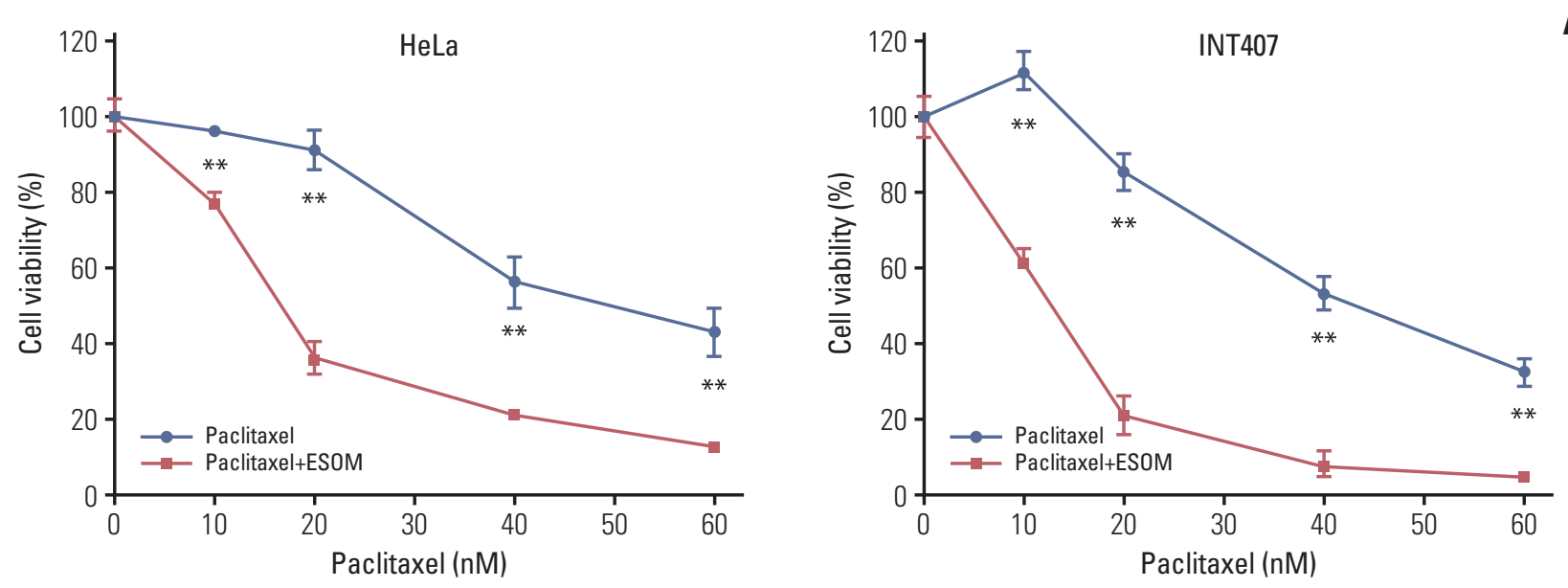

A

INT407
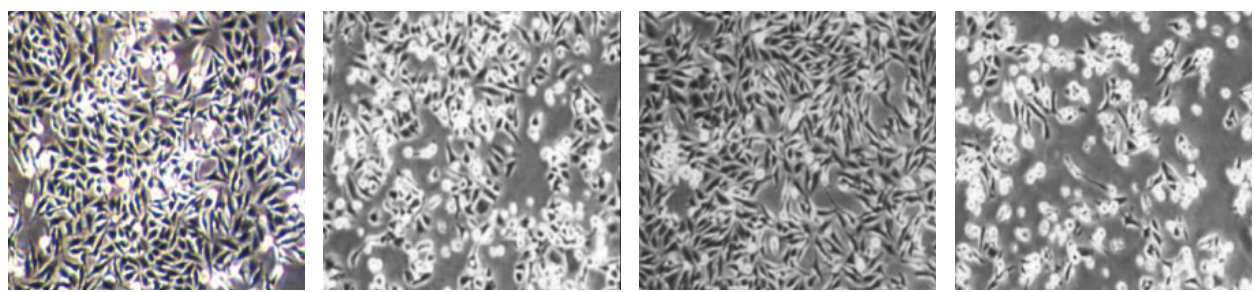

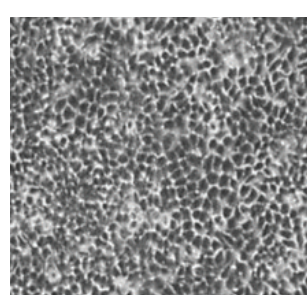

Control

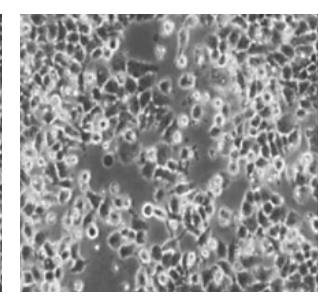

Paclitaxel

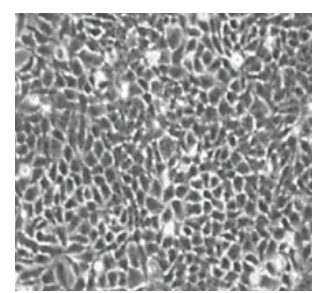

ESOM

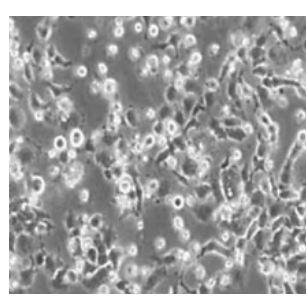

Paclitaxel+ESOM

C
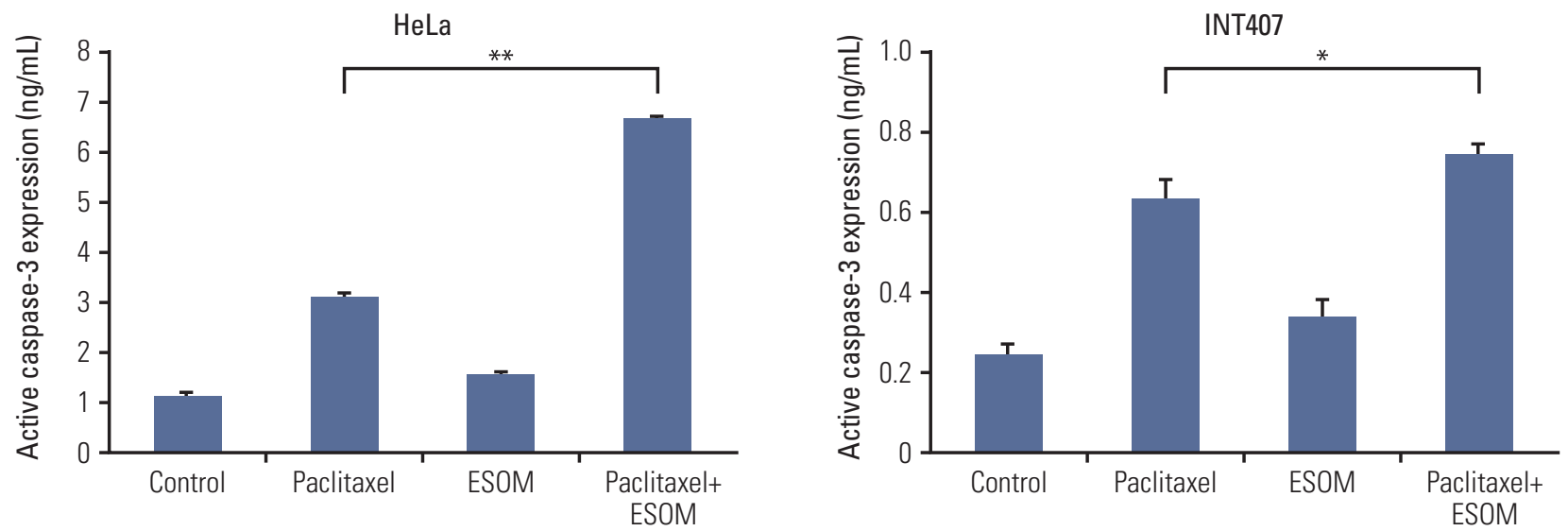

Fig. 4. Effects of esomeprazole (ESOM) pretreatment on cell survival and apoptosis with paclitaxel in HeLa and INT407 cells. (A) ESOM pretreatment significantly enhanced the cytotoxicity of paclitaxel in HeLa (37\%, p < 0.01) and INT407 (47\%, $\mathrm{p}<0.01$ ) cells when compared with paclitaxel treatment alone. (B) Cell death was observed by light microscopy in HeLa and INT407 cells $(\times 100)$. (C) Expression of active caspase-3 was measured by enzyme-linked immunosorbent assay in HeLa and INT407 cells. ${ }^{*} \mathrm{p}<0.05,{ }^{* *} \mathrm{p}<0.01$. 

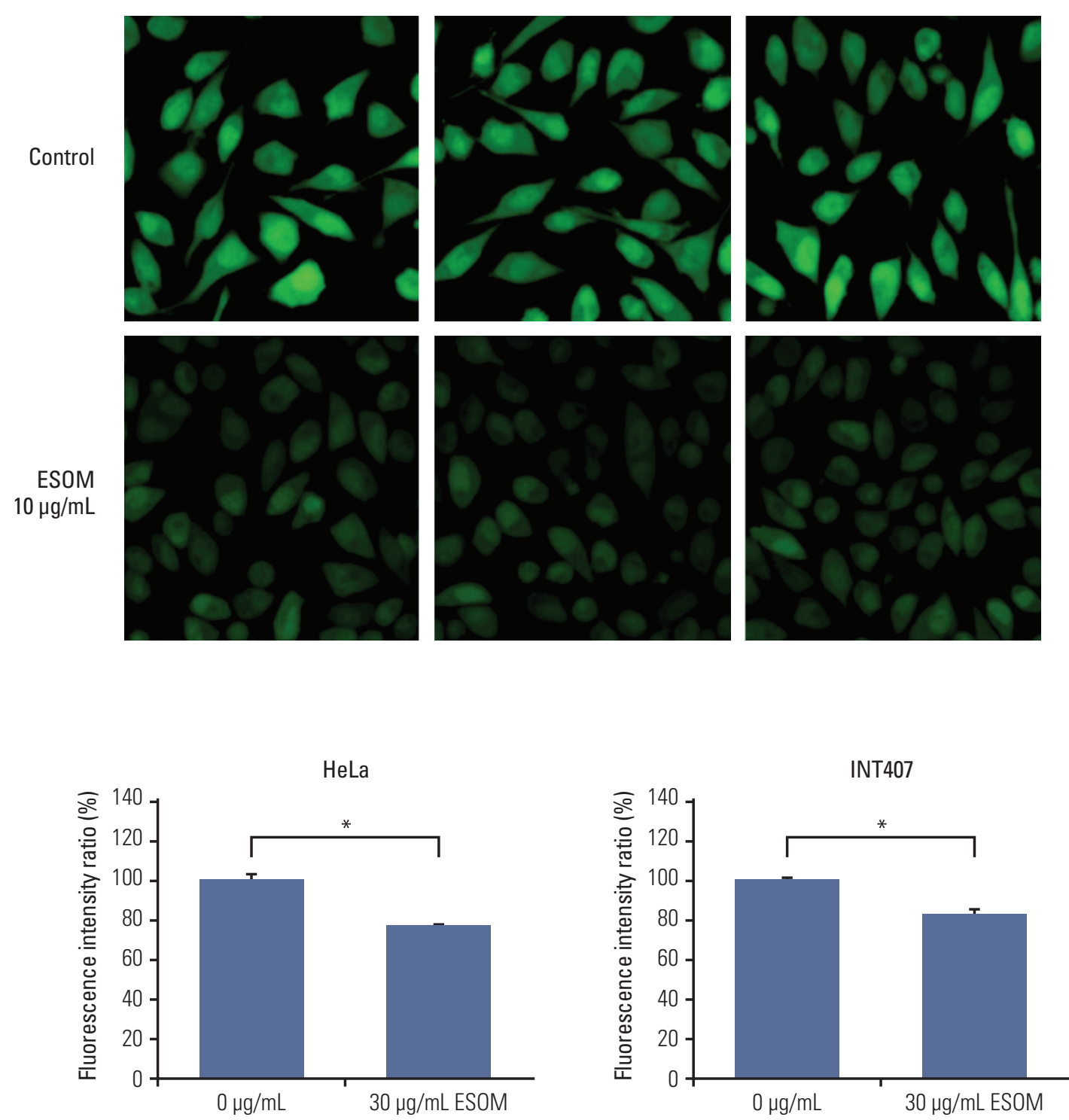

Fig. 5. Changes in intracellular pH. (A) Live-cell imaging of the BCECF intensity in HeLa cells with/without esomeprazole (ESOM) treatment using a LSM700 confocal microscope $(\times 400)$. (B) The cytosolic $\mathrm{pH}$ of HeLa and INT407 cells treated with $30 \mu \mathrm{g} / \mathrm{mL}$ ESOM for 3 hours decreased (both, $\mathrm{p}<0.01$ ). * $\mathrm{p}<0.05$.

poor prognosis in patients with bulky cervical tumor. Bulky solid tumors contain poorly perfused dense tumor tissue $[18,19]$, such as hypoxic tumors, in which protons and lactic acid are inefficiently removed from the extracellular space, creating an acidic extracellular microenvironment [20]. Therefore, proton pumps including V-ATPase are overexpressed to compensate for alkalization of the cytoplasm in the acidic microenvironment of the bulky hypoxic tumor $[21,22]$. However, when the activity of V-ATPase is inhibited using esomeprazole or its specific siRNA, the acidic $\mathrm{pH}$ of the tumor microenvironment is dramatically neutralized, which induces chemosensitization, inhibition of tumor proliferation, and apoptosis [22].

The results of the immunohistochemical analysis showed that the expression of V-ATPase in cervical adenocarcinomas was significantly higher than that of squamous cell cervical cancer $(73.4 \%$ vs. $57.1 \%, \mathrm{p}=0.016)$. This finding is consistent with those observed for non-small cell lung cancer [11]. The 
expression rate of V-ATPase was 83.7\% in lung adenocarcinoma, which was significantly higher than that of squamous cell lung cancer $(71.4 \%, \mathrm{p}<0.001)$ [11]. In clinical practice, cervical adenocarcinoma is commonly more resistant to chemotherapy drugs than squamous cell cervical cancer $[2,3]$. Furthermore, our in vitro study assessing the effects of esomeprazole pretreatment on cell survival after treatment with cytotoxic drugs demonstrated that the effects of esomeprazole pretreatment $(37 \%-47 \%)$ in HeLa and INT407 cells derived from adenocarcinoma were more remarkable than those (11\%-18\%) in SiHa and MS751 cells derived from non-adenocarcinomas such as squamous cell carcinoma (S4 Fig.). Together, these findings indicate that V-ATPase expression is potentially related to drug resistance in cervical adenocarcinoma.

PPIs such as esomeprazole and omeprazole, which are used clinically to suppress gastric acidity in peptic disease, are activated by acidic conditions. These inhibitors tend to decrease intracellular $\mathrm{pH}$ and increase extracellular $\mathrm{pH}$ via inhibition of V-ATPases through a covalent interaction [23]. PPIs have been shown to have anti-proliferative and proapoptotic effects on certain cancer cell lines, including B-cell leukemia, hepatoblastoma, melanoma, stomach cancer, sarcoma, and pancreatic cancer [24-27]. In the present study, while esomeprazole itself did not directly affect cell viability, it functioned as a chemosensitizer in cervical cancer cells. It remains unclear whether these findings are tumor specific or depend on tumor aggressiveness itself, regardless of tumor histology $[28,29]$. However, it is clear that modification of tumor acidity by blocking V-ATPase is a promising strategy for enhancing the sensitivity of the current cytotoxic treatments in cervical cancer.

Although the inhibition of V-ATPase via siRNA or PPI did not enhance the chemosensitivity of cisplatin in the present study, the results of a study performed by De Milito et al. [30] evaluating the effects of PPI pretreatment on human melanoma and osteosarcoma growth and cisplatin sensitivity in xenografted SCID/SCID mice differed. Specifically, they evaluated the effects of PPI pretreatment on human melanoma and osteosarcoma growth and cisplatin sensitivity in xenografted SCID/SCID mice and found this treatment sensitized tumor cell lines to the effects of cisplatin, resulting in decreases in the IC50 value by up to two-fold. Based on these findings, they concluded that oral pretreatment with PPI was able to induce/increase sensitivity of human solid tumors to cisplatin.

A search of clinical trial registries on April 8, 2015 yielded two phase II trials of PPI as a chemosensitizer in cancer patients undergoing chemotherapy registered at ClinicalTrials.gov. One was a completed trial on metastatic breast cancer (NCT01069081) that had not yet been published and another was an ongoing trial on castration-resistant prostate cancer (NCT01748500). Accordingly, further clinical trials investigating PPI in cervical cancer are needed.

It should be noted that this study had several limitations. Specifically, we did not validate the results of our study based on analysis of other PPIs such as omeprazole, lansoprazole, pantoprazole, or rabeprazole. We also did not perform an in vivo study to assess the potential clinical relevance of the in vitro results. However, positive aspects of this study include use of a large number of cancer tissue samples $(\mathrm{n}=351)$ with a long median follow-up period (65 months).

\section{Conclusion}

In conclusion, V-ATPase expression in cervical cancer was predominantly found in adenocarcinoma and was correlated with poor prognosis, especially in patients with bulky cervical tumor. Moreover, inhibition of the V-ATPase via PPI (esomeprazole) and its specific siRNA enhanced the sensitivity of chemotherapeutic agents in cervical cancer cell lines. These data suggest that a PPI may be useful as a chemosensitizer in treatment of patients with cervical cancer.

\section{Electronic Supplementary Material}

Supplementary materials are available at Cancer Research and Treatment website (http:// www.e-crt.org).

\section{Conflicts of Interest}

Conflict of interest relevant to this article was not reported.

\section{Acknowledgments}

This research was supported by a grant from the Korea Health Technology R\&D Project through the Korea Health Industry Development Institute (KHIDI), funded by the Ministry of Health \& Welfare, Republic of Korea (HI14C3418), and supported by Basic Science Research Program through the National Research Foundation of Korea (NRF), funded by the Ministry of Education (2013R1A1A2013612), as well 
as by a grant from the Korean Health Technology R\&D Project, Ministry for Health \& Welfare, Republic of Korea (HI14C1940). Additional funding was also received from the
National R\&D Program for Cancer Control, Ministry for Health, Welfare and Family Affairs, Republic of Korea (1520100).

\section{References}

1. Ferlay J, Shin HR, Bray F, Forman D, Mathers C, Parkin DM. Estimates of worldwide burden of cancer in 2008: GLOBOCAN 2008. Int J Cancer. 2010;127:2893-917.

2. Gien LT, Beauchemin MC, Thomas G. Adenocarcinoma: a unique cervical cancer. Gynecol Oncol. 2010;116:140-6.

3. Lee YY, Choi CH, Kim TJ, Lee JW, Kim BG, Lee JH, et al. A comparison of pure adenocarcinoma and squamous cell carcinoma of the cervix after radical hysterectomy in stage IB-IIA. Gynecol Oncol. 2011;120:439-43.

4. Peiretti M, Zapardiel I, Zanagnolo V, Landoni F, Morrow CP, Maggioni A. Management of recurrent cervical cancer: a review of the literature. Surg Oncol. 2012;21:e59-66.

5. Nishi T, Forgac M. The vacuolar $(\mathrm{H}+)$-ATPases: nature's most versatile proton pumps. Nat Rev Mol Cell Biol. 2002;3:94-103.

6. Martinez-Zaguilan R, Lynch RM, Martinez GM, Gillies RJ. Vacuolar-type $\mathrm{H}(+)$-ATPases are functionally expressed in plasma membranes of human tumor cells. Am J Physiol. 1993;265(4 Pt 1):C1015-29.

7. Mizunashi K, Furukawa Y, Katano K, Abe K. Effect of omeprazole, an inhibitor of $\mathrm{H}+, \mathrm{K}(+)$-ATPase, on bone resorption in humans. Calcif Tissue Int. 1993;53:21-5.

8. Sabolic I, Brown D, Verbavatz JM, Kleinman J. H(+)-ATPases of renal cortical and medullary endosomes are differentially sensitive to Sch-28080 and omeprazole. Am J Physiol. 1994; 266(6 Pt 2): F868-77.

9. Ha SY, Lee J, Jang J, Hong JY, Do IG, Park SH, et al. HER2-positive gastric cancer with concomitant MET and/or EGFR overexpression: a distinct subset of patients for dual inhibition therapy. Int J Cancer. 2015;136:1629-35.

10. Huang L, Lu Q, Han Y, Li Z, Zhang Z, Li X. ABCG2/V-ATPase was associated with the drug resistance and tumor metastasis of esophageal squamous cancer cells. Diagn Pathol. 2012;7:180.

11. Lu Q, Lu S, Huang L, Wang T, Wan Y, Zhou CX, et al. The expression of V-ATPase is associated with drug resistance and pathology of non-small-cell lung cancer. Diagn Pathol. 2013;8: 145.

12. De Milito A, Iessi E, Logozzi M, Lozupone F, Spada M, Marino $\mathrm{ML}$, et al. Proton pump inhibitors induce apoptosis of human B-cell tumors through a caspase-independent mechanism involving reactive oxygen species. Cancer Res. 2007;67: 5408-17.

13. Luciani F, Spada M, De Milito A, Molinari A, Rivoltini L, Montinaro A, et al. Effect of proton pump inhibitor pretreatment on resistance of solid tumors to cytotoxic drugs. J Natl Cancer Inst. 2004;96:1702-13

14. Boyer MJ, Tannock IF. Regulation of intracellular $\mathrm{pH}$ in tumor cell lines: influence of microenvironmental conditions. Cancer Res. 1992;52:4441-7.

15. Zhdanov AV, Dmitriev RI, Papkovsky DB. Bafilomycin A1 activates respiration of neuronal cells via uncoupling associated with flickering depolarization of mitochondria. Cell Mol Life Sci. 2011;68:903-17.

16. Lv C, Yang X, Yu B, Ma Q, Liu B, Liu Y. Blocking the Na+/H+ exchanger 1 with cariporide (HOE642) reduces the hypoxiainduced invasion of human tongue squamous cell carcinoma. Int J Oral Maxillofac Surg. 2012;41:1206-10.

17. Thomas GM. Improved treatment for cervical cancer: concurrent chemotherapy and radiotherapy. N Engl J Med. 1999;340: 1198-200.

18. Robova H, Rob L, Halaska MJ, Pluta M, Skapa P, Strnad P, et al. High-dose density neoadjuvant chemotherapy in bulky IB cervical cancer. Gynecol Oncol. 2013;128:49-53.

19. Yee GP, de Souza P, Khachigian LM. Current and potential treatments for cervical cancer. Curr Cancer Drug Targets. 2013;13:205-20.

20. Reshkin SJ, Greco MR, Cardone RA. Role of $\mathrm{pHi}$, and proton transporters in oncogene-driven neoplastic transformation. Philos Trans R Soc Lond B Biol Sci. 2014;369:20130100.

21. Boedtkjer E, Bunch L, Pedersen SF. Physiology, pharmacology and pathophysiology of the $\mathrm{pH}$ regulatory transport proteins NHE1 and NBCn1: similarities, differences, and implications for cancer therapy. Curr Pharm Des. 2012;18:1345-71.

22. Fais S, De Milito A, You H, Qin W. Targeting vacuolar H+-ATPases as a new strategy against cancer. Cancer Res. 2007;67: 10627-30.

23. Larsson H, Mattson H, Sundell G, Carlsson E. Animal pharmacodynamics of omeprazole: a survey of its pharmacological properties in vivo. Scand J Gastroenterol Suppl. 1985;108: 23-35.

24. Morimura T, Fujita K, Akita M, Nagashima M, Satomi A. The proton pump inhibitor inhibits cell growth and induces apoptosis in human hepatoblastoma. Pediatr Surg Int. 2008;24: 1087-94.

25. Yeo M, Kim DK, Kim YB, Oh TY, Lee JE, Cho SW, et al. Selective induction of apoptosis with proton pump inhibitor in gastric cancer cells. Clin Cancer Res. 2004;10:8687-96.

26. Yamagata M, Tannock IF. The chronic administration of drugs that inhibit the regulation of intracellular $\mathrm{pH}$ : in vitro and antitumour effects. Br J Cancer. 1996;73:1328-34.

27. Udelnow A, Kreyes A, Ellinger S, Landfester K, Walther P, Klapperstueck T, et al. Omeprazole inhibits proliferation and modulates autophagy in pancreatic cancer cells. PLoS One. 
2011;6:e20143.

28. Tredan O, Galmarini CM, Patel K, Tannock IF. Drug resistance and the solid tumor microenvironment. J Natl Cancer Inst. 2007;99:1441-54.

29. Spugnini EP, Citro G, Fais S. Proton pump inhibitors as anti vacuolar-ATPases drugs: a novel anticancer strategy. J Exp
Clin Cancer Res. 2010;29:44.

30. De Milito A, Luciani F, Fais S. How to overcome cisplatin resistance through proton pump inhibitors. In: Bonetti A, Leone R, Muggia FM, Howell SB, editors. Platinum and other heavy metal compounds in cancer chemotherapy. New York: Springer; 2009. p. 109-14. 